\title{
Integrating experimentation and quantitative modeling to enhance discovery of beta amyloid lowering therapeutics for Alzheimer's disease
}

\author{
Yasong $L u^{*}$ \\ Department of Pharmacokinetics, Dynamics and Metabolism, Pfizer Worldwide Research and Development, Groton, CT, USA
}

\author{
Edited by: \\ David Riddell, Pfizer, USA \\ Reviewed by: \\ Marcus Rattray, University of \\ Reading, UK \\ Jih-Heng Li, Kaohsiung Medical \\ University, Taiwan \\ *Correspondence: \\ Yasong Lu, Department of \\ Pharmacokinetics, Dynamics and \\ Metabolism, Pfizer Worldwide \\ Research and Development, Mail \\ Stop \#220-4546, Eastern Point Road, \\ Groton, CT 06340, USA. \\ e-mail: luyasong@yahoo.com
}

\begin{abstract}
Drug discovery can benefit from a proactive-knowledge-attainment philosophy which strategically integrates experimentation and pharmacokinetic/pharmacodynamic (PK/PD) modeling. Our programs for Alzheimer's disease (AD) illustrate such an approach. Compounds that inhibit the generation of brain beta amyloid $(A \beta)$, especially $A \beta 42$, are being pursued as potential disease-modifying therapeutics. Complexities in the PK/A $\beta$ relationship for these compounds have been observed and the data require an advanced approach for analysis. We established a semimechanistic PK/PD model that can describe the PK/A $\beta$ data by accounting for $A \beta$ generation and clearance. The modeling characterizes the in vivo PD (i.e., A $\beta$ lowering) properties of compounds and generates insights about the salient biological systems. The learning from the modeling enables us to establish a framework for predicting in vivo $A \beta$ lowering from in vitro parameters.
\end{abstract}

Keywords: $A \beta$, experimentation, quantitative modeling, efficiency, drug discovery

\section{INTRODUCTION}

The pharmaceutical industry has been experiencing a decrease in productivity despite increasing R\&D investment (FDA, 2004; Bunnage, 2011). Approaches for improving performance have been proposed from organizational (Sams-Dodd, 2005), operational (Bunnage, 2011; Johnstone et al., 2011; Knutsen, 2011; Elebring et al., 2012), and scientific perspectives (FDA, 2004; EMEA, 2007; Zhang et al., 2008; Morgan et al., 2012). Some researchers have argued that an increase in productivity may arise from a revamp of early discovery (Dimitri, 2011; Knutsen, 2011). Choosing the appropriate targets and optimal compounds in discovery should increase the probability of success at later stages (Bunnage, 2011; Maurer, 2011).

In the neuroscience area, Alzheimer's disease (AD) and dementia represent an urgent and significant unmet medical need. Drugs which temporarily alleviate some symptoms are on the market, but disease-modifying drugs which slow $\mathrm{AD}$ progression remain unavailable. While there are still uncertainties about AD etiology, the current leading hypothesis, known as amyloid cascade hypothesis (Hardy and Higgins, 1992), posits that AD is caused by an abnormal accumulation in the brain of amyloid beta $(\mathrm{A} \beta)$, a protein with a molecular weight of $\sim 4 \mathrm{kDa}$. $\mathrm{A} \beta$ is generated through sequential enzymatic cleavages of amyloid precursor protein, first by beta secretase (BACE1) and then by gamma secretase (GSI; reviewed in De Strooper et al., 2010). A $\beta$ has numerous forms depending on the exact cleavage site which dictates the length of the resulting amino acid sequence. $A \beta 40$ and $A \beta 42$ are the main forms found in the amyloid plaques in Alzheimer's brains (Gravina et al., 1995), and A $\beta 42$ is the predominant toxic form (El-Agnaf et al., 2000). Reduction of $A \beta$, particularly $A \beta 42$, in the brain therefore has been proposed as a potential disease-modifying treatment for AD. Potential therapies include small molecules that inhibit
BACE1 (BACEi) or gamma secretase (GSI) to lower total A $\beta$ production, or that modulate gamma secretase (GSM) to lower $A \beta 42$ selectively. All three approaches are being pursued in the pharmaceutical industry (reviewed in Ghosh et al., 2008; Imbimbo, 2008; Pettersson et al., 2011).

BACEi, GSI, or GSM programs seek compounds to test rigorously and definitely the amyloid cascade hypothesis in the clinic. Such compounds should be capable of distributing to the target site, interacting with the target, and eliciting sufficient pharmacodynamic (PD) response, i.e., $\mathrm{A} \beta$ lowering, in humans at concentrations that afford an acceptable safety margin. Preclinical identification of such compounds is based on intensive evaluation of pharmacokinetics (PK), PD, and safety in in vitro assays and preclinical animal models. This identification process is more efficient when the in vivo pharmacology and relevant biological systems are well understood.

A relevant in vivo measure of modulating secretase activities is brain $A \beta 42$ lowering, which in practice can be assessed only in preclinical species, typically rodents. In addition, CSF A $\beta 40$ and A $\beta 42$ are often monitored for their potential use as biomarkers for brain $A \beta$ lowering. Numerous data sets from in-house and external studies have demonstrated complexities in the PK/PD relationship for $A \beta$ lowering agents which pose challenges for both characterizing compounds' in vivo PD properties and translating effects across species. We have established a semimechanistically based PK/PD model to analyze $\mathrm{PK} / \mathrm{A} \beta$ data, and through its application have obtained reasonable characterization of compounds' in vivo $\mathrm{PD}$ properties and $\mathrm{A} \beta$ clearance kinetics (Wang et al., 2010; Lu et al., $2011,2012 a, b, c)$. Here, I summarize our systematic learning from quantitative modeling of the $A \beta$ data, and advocate for the integration of experimentation and PK/PD modeling using the BACEi, 
GSI, and GSM projects as an example. In this article, PD therefore refers to $A \beta$ lowering in the brain or CSF. Whether or not lowering brain $A \beta$ in patients will translate to clinical benefits is beyond the scope of this article.

\section{COMPLEXITIES IN PK/A $\beta$ DATA}

The relationship between the PK and $A \beta$ data for BACEi, GSI, and GSM is complex. First, $A \beta$ lowering after compound treatment shows hysteresis (Figures 1A,B; Hawkins et al., 2011; Lu et al., 2011, 2012c), a tendency for an effect profile to lag temporally behind an exposure profile. Plotting $A \beta$ levels vs. the concurrent exposures yields a hysteresis loop; the effect does not correlate strictly with concentration, and instead also depends on time (as can be seen in Figure 1B). Second, within a given species (mouse, rat, or guinea pig), the data from single-time-point sampling often show stronger $A \beta$ lowering in CSF than in brain with the discrepancy widening as the dose increases (Figure 1C; Wang et al., 2010; Lu et al., 2012c). Third, following dosing the time courses of $\mathrm{CSF}$ and brain $\mathrm{A} \beta$ diverge from one another. Figure 1A illustrates this behavior observed in the mouse, rat, and guinea pig; CSF $\mathrm{A} \beta$ decreases and returns to baseline more rapidly than brain $\mathrm{A} \beta$ (Lu et al., 2011,2012c). The separation is increasingly pronounced with dose (Wang et al., 2010). Fourth, the shape of the CSF A $\beta$ profile varies across species. The shape becomes more blunted with increasing body size (Figure 1D). These observations provoke a series of critical questions:

- How should we appropriately characterize a compound's in vivo PD properties (potency and efficacy)?

- Why are there differences in the effect size and temporal profile between brain and CSF and across species?

- Is CSF $A \beta$ a valid biomarker for brain $A \beta$ lowering given the discrepancy in $A \beta$ lowering between the two compartments?

- Are the mouse and rat suitable pharmacology models for humans, and if yes, how should we scale an $\mathrm{A} \beta$ lowering effect from these species to humans?

Each question represents a substantial hurdle for rational and efficient discovery. It is therefore critical to seek a sound mechanistic understanding of the complexities and obtain answers to these questions.

\section{A SEMIMECHANISTICALLY BASED PK/PD MODEL FOR ANALYZING A $\beta$ DATA}

The hysteresis precludes the use of the classical sigmoidal model which assumes that the PD results from the concurrent drug concentration. A more sophisticated model is thus necessary. We established a semimechanistic model that can describe the complex $\mathrm{PK} / \mathrm{A} \beta$ data by taking $\mathrm{A} \beta$ generation and clearance into consideration (Lu et al., 2011, 2012a,c). As shown in Figure 1E, this model assumes that the level of steady-state $A \beta$ in a compartment is maintained via the balancing of a zero-order generation rate $\left(\mathrm{K}_{\mathrm{in}}\right)$ and a first-order clearance process (with a fractional turnover rate of $k_{\text {out }}$ ). Consistent with the mode of action of BACEi, GSI, or GSM, an inhibitory effect is described by a sigmoidal term that modifies the generation rate $\mathrm{K}_{\mathrm{in}}$ (Eq. 1). The drug concentration (C) may be that in plasma, brain, or CSF depending upon the data available. This model represents a biologically reasonable simplification of $\mathrm{A} \beta$ homeostasis and its pharmacological modulation.

$$
\frac{d A \beta}{d t}=K_{\text {in }} \times\left(1-\frac{I_{\max } \times C^{\gamma}}{I C_{50} \gamma+C^{\gamma}}\right)-k_{\text {out }} \times A \beta
$$

To remove potential confounding non-specific effects, the absolute $A \beta$ levels in treatment groups are expressed as percentages of the concurrent vehicle control and the normalized time courses are then modeled. By fitting the normalized data, the model produces estimates for the unknown parameters: $k_{\text {out }}, I_{\max }$ (maximum inhibition of $K_{\text {in }}$ ), $\mathrm{IC}_{50}$ (concentration at which $50 \%$ of $I_{\max }$ is achieved), and $\gamma$ (Hill coefficient). At steady-state, Eq. 1 simplifies to $K_{\text {in }}=100 \% \times k_{\text {out }}$ since in this scenario the $\mathrm{A} \beta$ level is constant at $100 \%$ of the basal level. Once $k_{\text {out }}$ is estimated, $K_{\text {in }}$ can be readily calculated.

\section{INSIGHTS FROM PK/PD MODELING}

The PK/PD modeling extracts, from complex PK/A $\beta$ data, parameters that allow appropriate characterization of the properties of compounds' in vivo pharmacology and the pertinent biological systems.

\section{CHARACTERIZATION OF A COMPOUND'S IN VIVO PD PROPERTIES}

A compound's in vivo potency and efficacy for lowering $\mathrm{A} \beta$ can be defined by the modeling-derived $\mathrm{IC}_{50}$ and $I_{\max }$, respectively. For estimation of steady-state average $\mathrm{A} \beta$ lowering after treatment, a relationship between exposure and the modified $\mathrm{A} \beta$ generation rate, $R_{\text {gen }}$ as a fraction of the control is defined by Eq. 2

$R_{\text {gen }}=\frac{K_{\text {in }} \times\left(1-\frac{I_{\max } \times C^{\gamma}}{I C_{50} C^{\gamma}}\right)}{K_{\text {in }}}=1-\frac{I_{\max } \times C^{\gamma}}{I C_{50} \gamma+C^{\gamma}}$

This exposure $-R_{\text {gen }}$ relationship defines the intrinsic PK/PD relationship for a BACEi, GSI, or GSM. It is devoid of confounds arising from PK behaviors or $\mathrm{A} \beta$ turnover kinetics. Mathematically, this intrinsic $\mathrm{PK} / \mathrm{PD}$ relationship is equivalent to the relationship between an exposure and time-weighted-average $A \beta$ lowering at steady-state after repeated dosing.

Empirical non-modeling based analyses of PK/A $\beta$ data may involve several approaches, such as single-time-point exposure/A $\beta$ assessment, area-under-the-concentration-curve (AUC) vs. maximum $A \beta$ lowering, or AUC vs. area-under-the-A $\beta$-curve assessment. As discussed earlier (Lu et al., 2011), these approaches have serious flaws and limitations. They not only lack necessary predictive or extrapolating power, but also likely yield misleading or erroneous potency estimates for a compound. Therefore, for the discovery of $A \beta$ lowering therapeutics, these approaches should be replaced by quantitative $\mathrm{PK} / \mathrm{PD}$ modeling.

\section{CHARACTERIZATION OF PERTINENT BIOLOGICAL SYSTEMS}

The biological systems involved in the discovery of $A \beta$ lowering therapeutics include in vitro assays for high throughput screening and animal models such as mice, rats, guinea pigs, dogs, monkeys, or humans for in vivo pharmacology evaluation. 

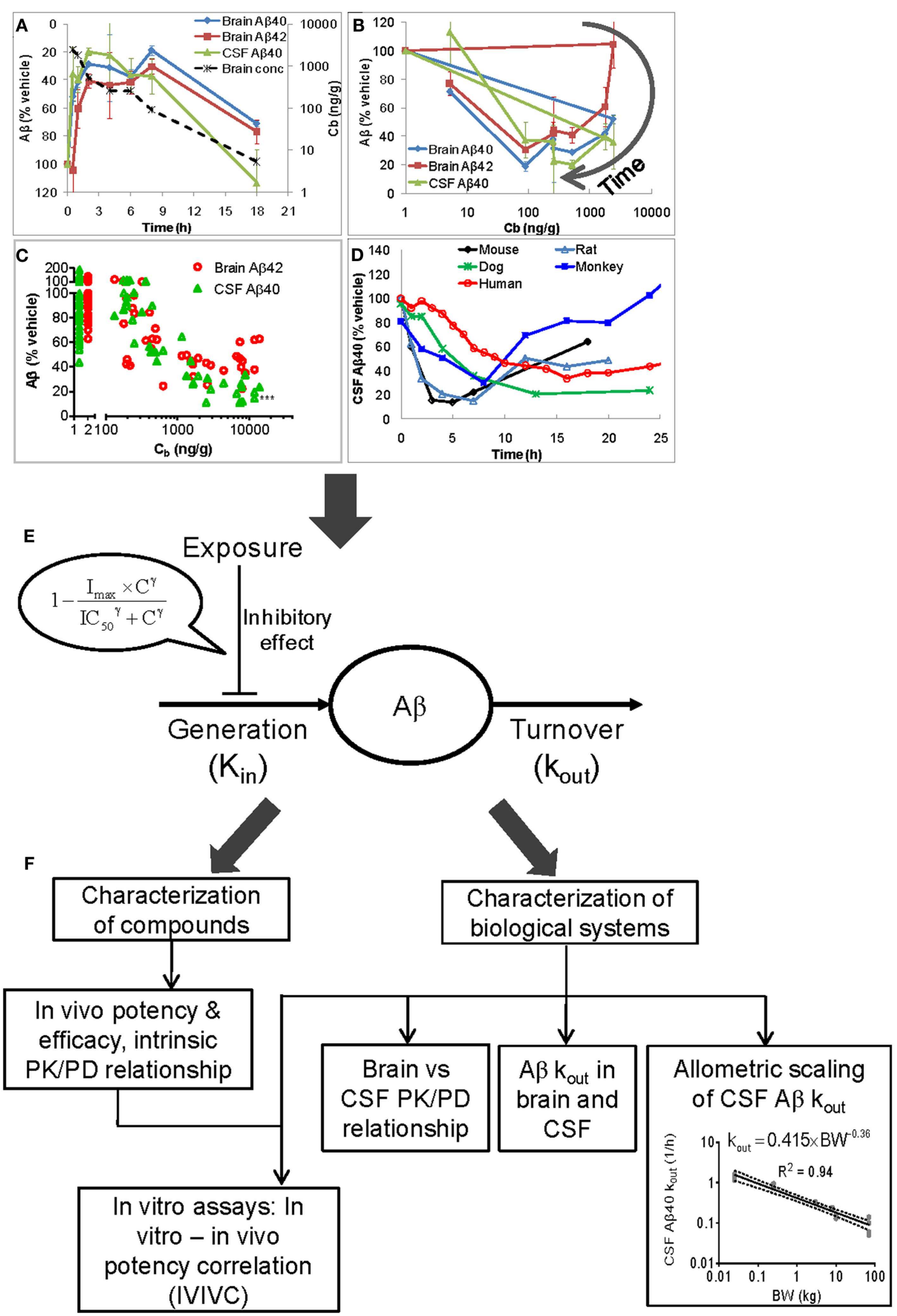

FIGURE 1 | Continued 


\section{FIGURE 1 | Inherent complexities in PK/A $\beta$ data (A-D), the} semimechanistically based PK/PD model (E) for analyzing $A \beta$ data, and the insights from the modeling (F). The complexities in the data are reflected by hysteresis $(\mathbf{A}, \mathbf{B})$, differences in the effect size between brain and $\operatorname{CSF}(\mathbf{A}, \mathbf{C})$ and in the temporal profiles for brain and CSF $A \beta(\mathbf{A})$, and variation of CSF A $\beta$ temporal profile across species (D). (A): Time course data from 129/SVE mice treated orally with LY450139 (GSI) at $150 \mathrm{mg} / \mathrm{kg}$ (Lu et al., 2011). The effect time courses lag temporally behind the brain concentration time course. (B) The time course data in (A) plotted as $A \beta$ vs. exposure to illustrate the hysteresis loops, where the effect does not correlate strictly with drug concentration, and instead also depends on time. (A,B) suggest a delay between drug concentration and manifestation of an effect, a phenomenon known as hysteresis. (C) Data at $3 \mathrm{~h}$ post-dosing from 129/SVE mice treated subcutaneously with LY2811376 (BACEi) at 1, 3, 10,30, and $100 \mathrm{mg} / \mathrm{kg}$ (reproduced from Lu et al., 2012c with permission from American Society for Pharmacology and Experimental Therapeutics). Each symbol represents an individual animal. The individuals in the $1-2 \mathrm{ng} / \mathrm{g}$ range are vehicle controls, adjusted from the actual concentration of zero for illustration on the logarithmic scale. (D) The mean time course profiles of CSF A $\beta 40$ in the 129/SVE mouse, Sprague-Dawley rat, cynomolgus monkey, beagle dog, and healthy human subject treated with LY2811376 at $100 \mathrm{mg} / \mathrm{kg}$, subcutaneously,
$50 \mathrm{mg} / \mathrm{kg}$, orally, $20 \mathrm{mg} / \mathrm{kg}$, orally, $5 \mathrm{mg} / \mathrm{kg}$, orally, and $90 \mathrm{mg}$, orally, respectively. (E) The semimechanistic model assumes that the $A \beta$ level is controlled by a zero-order generation rate, which is modified by an inhibitory effect due to BACEi, GSI, or GSM, and a first-order clearance process. (F) The modeling enables characterization of compounds' in vivo PD properties and the relevant biological systems. The plot of allometric scaling of CSF A $\beta 40 k_{\text {out }}$ is reproduced from (Lu et al., 2012a) with permission from S. Karger AG, Basel, Switzerland, and updated with inclusion of the rat. By reversing the directions of all arrows, this figure illustrates an integrative framework for projecting compounds' in vivo PD behaviors from in vitro and system parameters. Hysteresis: A tendency for an effect profile to lag temporally behind an exposure profile after drug treatment. Plotting $A \beta$ levels vs. the concurrent exposures yields a hysteresis loop; the effect does not correlate strictly with concentration, and instead also depends on time. Hysteresis demonstrates an apparent lack of exposure-response relationship. Analysis of PK/PD data with hysteresis requires complex models, such as a link model, an indirect response model, or a mechanistically based model (Mager et al., 2003; Danhof et al., 2007). Cb, drug brain concentration; $K_{\text {in }}$ A $\beta$ generation rate; $k_{\text {out }}$, first-order rate constant for $A \beta$ clearance; $I_{\max }$, maximum inhibition of $K_{\text {in }} ; I_{50}$, concentration that causes half-maximum inhibition of $K_{\text {in }} ; \gamma$, Hill coefficient; BW, body weight.

\section{In vitro assays}

An in vitro assay provides a measure of potency $\left(\mathrm{IC}_{50}\right)$, which is one of the primary in vitro parameters for directing synthesis of new compounds and for triaging, rank ordering, and prioritizing among existing compounds. Before an in vitro assay can be relied upon, a validation from the translational pharmacology standpoint has to be conducted to assess how the in vitro potency is correlated with in vivo potency. With in vivo potency estimated by $\mathrm{PK} / \mathrm{PD}$ modeling, an in vitro - in vivo potency correlation (IVIVC) may be established. The IVIVC will allow selection of the most relevant in vitro assay and determination of the quantitative in vitro - in vivo translation.

\section{Animal models}

Through PK/PD modeling of extensive data sets, we have determined the turnover rate $\left(k_{\text {out }}\right)$ for $\mathrm{A} \beta$ in the brain and CSF in the mouse (non-transgenic), rat, and guinea pig. The $k_{\text {out }}$ in brain is slower than in CSF in all three species. In the mouse, the $k_{\text {out }}$ for brain $A \beta 42$ is approximately threefold lower than that for CSF $\mathrm{A} \beta 40$ (0.49 vs. 1.42/h; Lu et al., 2012a), and in the rat and guinea pig, the $k_{\text {out }}$ for brain $\mathrm{A} \beta 42$ is about twofold lower than that for CSF A $\beta 42$ (Lu et al., 2012c). The difference in the $k_{\text {out }}$ leads to distinct $A \beta$ profiles and effect sizes at a given sampling point in the two compartments following treatment (Lu et al., 2012a).

We have also determined the $k_{\text {out }}$ for CSF A $\beta 40$ in the rat, dog, monkey, and human in addition to the mouse. The $k_{\text {out }}$ value scales allometrically across these five species, following the equation $k_{\text {out }}=0.415 \times \mathrm{BW}^{-0.36}$, where $\mathrm{BW}$ represents the body weight (Figure 1F). The decrease in the $k_{\text {out }}$ with body weight causes the increasingly blunted shapes of the CSF A $\beta 40$ time course (Lu et al., 2012a).

CSF $A \beta$ is thought to be primarily derived from the brain. As long as the $\mathrm{PK} / \mathrm{PD}$ relationship for $\mathrm{A} \beta$ lowering in CSF is consistently related to that in brain, CSF $A \beta$ may serve as a biomarker for brain $A \beta$ lowering despite the discrepancy in $A \beta$ profiles in the two compartments. Our modeling analyses of seven compounds, across the three mechanisms (BACEi, GSI, and GSM), in three species (mouse, rat, and guinea pig) demonstrated a consistent overlap of the intrinsic PK/PD relationships for brain and CSF over the range of $0-50 \%$ lowering in $R_{\text {gen }}$ (Lu et al., 2012c). This analysis supports CSF $A \beta$ as a potential biomarker for brain $A \beta$ lowering from the clinical trial standpoint.

Another conundrum that modeling can shed light on is which preclinical species are suitable pharmacology models for evaluating BACEi, GSI, and GSM. The choice is generally a compromise between multiple factors, such as animal cost (direct cost, husbandry, and genotyping if applicable), animal size (relevant to chemical scale up), homology of $A \beta$ sequence (human shares the same sequence with the guinea pig, dog, and monkey, but not mice or rats), streamlined operation (preferentially the same species for pharmacology evaluation, absorption, distribution, metabolism, and elimination profiling, and safety characterization), and predictivity for human $A \beta$ lowering. The predictivity for human $A \beta$ lowering can be assessed based on interspecies comparisons of the intrinsic $\mathrm{PK} / \mathrm{PD}$ relationship. When the interspecies translation is understood, a rational choice of the suitable model may be made. In this regard, two examples have been reported. (1) The transgenic Tg2576 mouse (Hsiao et al., 1996) has been recognized as a model for AD, and traditionally has been used to identify GSI despite the tremendous cost. Since the primary PD endpoint for GSI is A $\beta$ lowering in the central compartments, a non-transgenic strain may be suitable if the PK/PD relationship in this strain is predictive of that in humans. Therefore, we undertook comprehensive PK/PD analyses for several GSI compounds, including LY450139 and BMS-708163, in the Tg2576 mouse, non-transgenic 129/SVE mouse, rat, guinea pig, dog, monkey, and human. Our analyses indicated that the intrinsic $\mathrm{PK} / \mathrm{PD}$ relationship for brain $\mathrm{A} \beta 42$ and CSF $A \beta 40$ in the 129/SVE mouse is consistent with, and hence predictive of, that observed for CSF A $\beta$ in humans (Lu et al., 2011). As a result, we suggested the 129/SVE mouse as a reasonable model for the discovery of GSI (Lu et al., 2011), allowing for a drastic reduction in the cost on animals. (2) For identification 
of GSM, the guinea pig was thought to be a preferred model due to its $A \beta$ sequence being identical to that of human. This choice was not ideal as guinea pigs are generally not used for PK or safety profiling. To address the conflict, we collected PK/A $\beta$ data for two structurally distinct GSM in the two species. Our modeling analyses demonstrated a similar intrinsic PK/PD relationship for $\mathrm{A} \beta 42$ lowering in the central compartments in guinea pig and rat (Lu et al., 2012b). Although the predictivity of these species for humans remains unknown due to lack of available clinical data, this result suggests that the difference in the $A \beta$ sequence has no bearing on the PD response and thus justifies the substitution of rats for guinea pigs for GSM evaluation.

\section{USES OF PK/PD MODELING AND SIMULATION}

An $A \beta$ time course profile is determined by three components, exposure time course, intrinsic $\mathrm{PK} / \mathrm{PD}$ relationship, and $\mathrm{A} \beta$ turnover rate (Lu et al., 2011). Once understood, these components may be re-integrated using a PK/PD model to predict the time course of $A \beta$ concentrations after a treatment (Lu et al., 2012a,c). This exercise has several advantages for discovery research. First, it may replace studies that do not necessarily generate new information. For example, if a compound is characterized for its PK/PD relationship for $A \beta$ lowering at 5 and $30 \mathrm{mg} / \mathrm{kg}$ subcutaneously, one may later be interested in its effects at $10 \mathrm{mg} / \mathrm{kg}$ orally. With an oral PK time course at $10 \mathrm{mg} / \mathrm{kg}$, along with the intrinsic PK/PD relationship and $\mathrm{A} \beta$ turnover rate derived earlier, a PD profile can be predicted from simulations (see an example in Figure 2; the data set not used in the model development is predicted adequately by the model.) As such, the oral PD time course study is obviated. Second, it can guide design of preclinical studies and clinical trials. The predictions of $A \beta$ time courses facilitate selections of appropriate doses and sampling time points to ensure the outcomes are informative. We have been using this approach routinely to design our studies in multiple preclinical species. The high quality of the data from such studies has been appreciated internally. Third, modeling and simulation is a useful tool for hypothesis testing. While preclinical PK/PD characterization is often conducted in an acute, single-dose setting, a chronic, repeated-dosing regimen is generally clinically relevant. It is therefore necessary to assess whether the PK/PD relationship shifts after repeated dosing. With the null hypothesis of no shift, the simulations of $A \beta$ profiles after chronic treatment are then compared with observations from a subsequent chronic preclinical study. The agreement or disagreement between the two allows inference of failure to reject the null hypothesis or not. These applications of the modeling and simulation ensure a return of informative data and knowledge on a given resource investment.

\section{SUFFICIENT EXPERIMENTAL DATA TO ENABLE PARAMETER ESTIMATION}

The utility of PK/PD modeling and simulation is contingent on reliable estimation of the unknown parameters, $k_{\text {out }}, I_{\max }, \mathrm{IC}_{50}$, and $\gamma$, which, in turn, is driven by sufficiently informative data. Note that "sufficiently informative data" is fundamentally different from "lots of data;" rather, it is characterized by wide coverage of the dynamic range (from minimal to near maximum lowering of $\mathrm{A} \beta$ ) and ample time course sampling points (capturing the

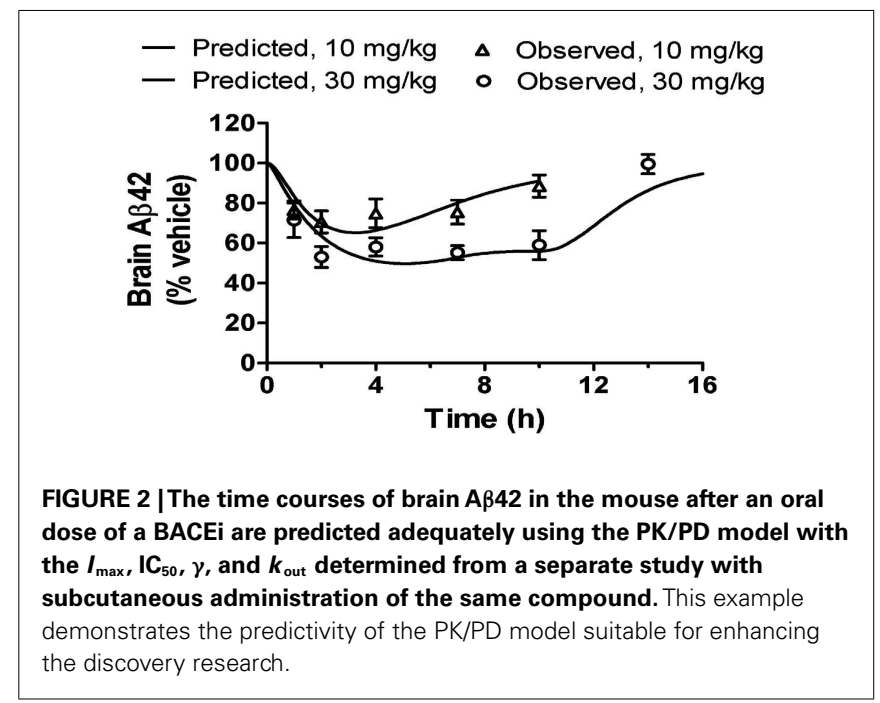

effect onset and offset phases). Single-time-point dose - response studies, commonly conducted for the BACEi, GSI, and GSM programs in the industry, carry less information because they only capture a snapshot of whole time courses. In our practice, we have de-prioritized dose - response studies and directed resources to well-designed time course studies (Wang et al., 2010; Lu et al., 2011, 2012a,b,c). While time course studies appear to require more resources, we believe that the value that these studies bring warrants the investment.

\section{REACTIVE DATA COLLECTION VS. PROACTIVE-KNOWLEDGE-ATTAINMENT}

It is common that a discovery process is driven by identification of key compounds. That is, once meeting certain criteria, a compound is fully profiled for PK and PD properties. If a detrimental liability is later identified, the compound, along with all the relevant data, is then abandoned. As soon as another compound hits the criteria, the next cycle starts. In this process, data are collected reactive to the identification of presumably viable compounds, and little systematic knowledge is accumulated along the way. The insights from and uses of modeling discussed above stem from the proactive-knowledge-attainment philosophy implemented in our BACEi, GSI, and GSM programs. With this philosophy, a discovery program can be executed in two phases: accumulating necessary systematic knowledge using compounds of diverse properties irrespective of their druggability, and applying the knowledge to guide lead optimization, compound selection, and pharmacological translation to the clinic. This strategy is likely to enhance the efficiency of discovery research, and is of similar spirit to the proposals by other researchers (Mager et al., 2009; Maurer, 2011; van der Graaf and Benson, 2011).

\section{SUMMARY}

Small molecule BACEi, GSI, and GSM have been pursued as potential disease-modifying drugs for $\mathrm{AD}$. The preclinical pharmacological activities of these compounds are assessed primarily with the reduction in brain and CSF A $\beta$. Complexities in the PK/A $\beta$ data for these compounds have been observed, and require improved 
approaches for analysis. Using a semimechanistically based PK/PD model, we are able to characterize reasonably the in vivo PD properties of compounds and the relevant biological systems. This characterization enables establishment of an integrative framework for predicting a compound's in vivo PD behaviors from in vitro parameters. The proactive-knowledge-attainment philosophy has driven the research operations of our BACEi, GSI, and GSM programs. With intensive experimentation and modeling analyses, we have achieved a plausible mechanistic understanding of the apparent discrepancy of $A \beta$ lowering profiles across compartments and across species, and have been able to choose

\section{REFERENCES}

Bunnage, M. E. (2011). Getting pharmaceutical R\&D back on target. Nat. Chem. Biol. 7, 335-339.

Danhof, M., de Jongh, J., De Lange, E. C., Della Pasqua, O., Ploeger, B. A., and Voskuyl, R. A. (2007). Mechanismbased pharmacokinetic-pharmacodynamic modeling: biophase distribution, receptor theory, and dynamical systems analysis. Annu. Rev. Pharmacol. Toxicol. 47, 357-400.

De Strooper, B., Vassar, R., and Golde, T. (2010). The secretases: enzymes with therapeutic potential in Alzheimer disease. Nat. Rev. Neurol. 6, 99-107.

Dimitri, N. (2011). An assessment of R\&D productivity in the pharmaceutical industry. Trends Pharmacol. Sci. 32, 683-685.

El-Agnaf, O. M., Mahil, D. S., Patel, B. P., and Austen, B. M. (2000). Oligomerization and toxicity of beta-amyloid42 implicated in Alzheimer's disease. Biochem. Biophys. Res. Commun. 273, 1003-1007.

Elebring, T., Gill, A., and Plowright, A. T. (2012). What is the most important approach in current drug discovery: doing the right things or doing things right? Drug Discov. Today. doi: 10.1016/j.drudis.2012.04.009

EMEA. (2007). Innovative Drug Development Approaches: Final Report from the European Medicines Agency/CHMP Think-Tank Group on Innovative Drug Development. Report number EMEA/127318/2007. London: European Medicines Agency.

FDA. (2004). Innovation or Stagnation: challenge and Opportunity on the Critical Path to New Medical Products. Available at: http://www.fda. gov/downloads/ScienceResearch/ SpecialTopics/CriticalPathInitiative/ CriticalPathOpportunitiesReports/ ucm113411.pdf (accessed June 19, 2012).

Ghosh, A. K., Gemma, S., and Tang, J. (2008). Beta-secretase as a therapeutic target for Alzheimer's disease. Neurotherapeutics 5, 399-408.
Gravina, S. A., Ho, L., Eckman, C. B. Long, K. E., Otvos, L. Jr., Younkin, L. H., et al. (1995). Amyloid beta protein (A beta) in Alzheimer's disease brain. Biochemical and immunocytochemical analysis with antibodies specific for forms ending at A beta 40 or A beta 42(43). J. Biol. Chem. 270, 7013-7016.

Hardy, J. A., and Higgins, G. A. (1992). Alzheimer's disease: the amyloid cascade hypothesis. Science 256, 184-185.

Hawkins, J., Harrison, D. C., Ahmed, S., Davis, R. P., Chapman, T., Marshall, I., et al. (2011). Dynamics of Abeta42 reduction in plasma, CSF and brain of rats treated with the gamma-secretase modulator, GSM-10h. Neurodegener. Dis. 8, 455-464.

Hsiao, K., Chapman, P., Nilsen, S., Eckman, C., Harigaya, Y., Younkin, S., et al. (1996). Correlative memory deficits, Abeta elevation, and amyloid plaques in transgenic mice. Science 274, 99-102.

Imbimbo, B. P. (2008). Therapeutic potential of gamma-secretase inhibitors and modulators. Curr.

Johnstone, C., Pairaudeau, G., and Pettersson, J. A. (2011). Creativity, innovation and lean sigma: a controversial combination? Drug Discov. Today 16, 50-57.

Knutsen, L. J. (2011). Drug discovery management, small is still beautiful: why a number of companies get it wrong. Drug Discov. Today 16, 476-484.

Lu, Y., Barton, H. A., Leung, L., Zhang, L., Hajos-Korcsok, E., Nolan, C. E., et al. (2012a). Cerebrospinal fluid A $\beta$ turnover in the mouse, dog, monkey, and human evaluated by systematic quantitative analyses. Neurodegener. Dis. doi: 10.1159/000341217

Lu, Y., Wood, K. M., Steyn, S. J., Becker, S. L., Osgood, S. M., Miller, E. H., et al. (2012b). "Gamma secretase modulators demonstrate similar exposureresponse relationship in the rat Top. Med. Chem. 8, 54-61.

suitable species, e.g., non-transgenic over $\operatorname{Tg} 2576$ mouse, rat over guinea pig, for preclinical in vivo assessment. Given the values it has brought, this proactive-knowledge-attainment philosophy is expected to enhance our ability to select high quality compounds for clinical testing.

\section{ACKNOWLEDGMENTS}

The author is grateful to Drs. Hugh A. Barton and Patrick Trapa at the Department of Pharmacokinetics, Dynamics and Metabolism, Pfizer Worldwide Research and Development, and the reviewers for their critiques.

and guinea pig," in Poster Presentation at Alzheime's Association International Conference (AAIC, 2012), Vancouver.

Lu, Y., Zhang, L., Nolan, C. E., Riddell, D., Hajos-Korcsok, E., Bales, K. et al. (2012c). Cerebrospinal fluid amyloid-beta (Abeta) as an effect biomarker for brain Abeta lowering verified by quantitative preclinical analyses. J. Pharmacol. Exp. Ther. 342, 366-375.

Lu, Y., Zhang, L., Nolan, C. E., Becker, S. L., Atchison, K., Robshaw, A. E., et al. (2011). Quantitative pharmacokinetic/pharmacodynamic analyses suggest that the 129/SVE mouse is a suitable preclinical pharmacology model for identifying small-molecule gamma-secretase inhibitors. J. Pharmacol. Exp. Ther. 339, 922-934.

Mager, D. E., Woo, S., and Jusko, W. J. (2009). Scaling pharmacodynamics from in vitro and preclinical animal studies to humans. Drug Metab. Pharmacokinet. 24, 16-24.

Mager, D. E., Wyska, E., and Jusko, W. J. (2003). Diversity of mechanismbased pharmacodynamic models. Drug Metab. Dispos. 31, 510-518.

Maurer, T. (2011). Translational Modeling \& Simulation in Support of Rational Drug Discovery: principles, Challenges and Examples. Rosa's WorldWide Webinars, 2/1/2012. Seattle: Rosa \& Co. LLC.

Morgan, P., Van Der Graaf, P. H. Arrowsmith, J., Feltner, D. E., Drummond, K. S., Wegner, C. D., et al. (2012). Can the flow of medicines be improved? Fundamental pharmacokinetic and pharmacological principles toward improving phase II survival. Drug Discov. Today 17, 419-424.

Pettersson, M., Kauffman, G. W., am Ende, C. W., Patel, N. C., Stiff, C., Tran, T. P., et al. (2011). Novel gamma-secretase modulators: a review of patents from 2008 to 2010. Expert Opin. Ther. Pat. 21, 205-226.
Sams-Dodd, F. (2005). Optimizing the discovery organization for innovation. Drug Discov. Today 10 , 1049-1056.

van der Graaf, P. H., and Benson, N. (2011). Systems pharmacology: bridging systems biology and pharmacokineticspharmacodynamics (PKPD) in drug discovery and development. Pharm. Res. 28, 1460-1464.

Wang, E. Q., Tseng, E., Lu, Y., Becker, S. L., Hudson, E., Zhang, L., et al. (2010). "Pharmacokinetic/pharmacodynamic analysis of Beta-Amyloid response following modulation of the gamma secretase enzyme," in Poster Presentation at the International Conference on Alzheimer's Disease (ICAD), Honolulu, HI.

Zhang, L., Pfister, M., and Meibohm, B. (2008). Concepts and challenges in quantitative pharmacology and model-based drug development. AAPS J. 10, 552-559.

Conflict of Interest Statement: Yasong Lu was an employee of Pfizer Inc. when preparing this article.

Received: 24 June 2012; accepted: 14 September 2012; published online: 04 October 2012.

Citation: Lu Y (2012) Integrating experimentation and quantitative modeling to enhance discovery of beta amyloid lowering therapeutics for Alzheimer's disease. Front. Pharmacol. 3:177. doi: 10.3389/fphar.2012.00177

This article was submitted to Frontiers in Experimental Pharmacology and Drug Discovery, a specialty of Frontiers in Pharmacology.

Copyright (c) $2012 \mathrm{Lu}$. This is an openaccess article distributed under the terms of the Creative Commons Attribution License, which permits use, distribution and reproduction in other forums, provided the original authors and source are credited and subject to any copyright notices concerning any third-party graphics etc. 